\title{
Pitfalls and mimics: The many facets of normal paediatric thymus
}

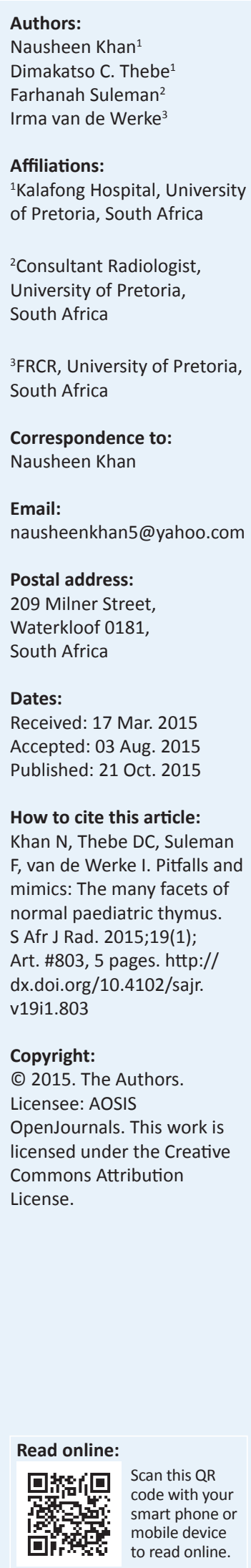

The thymus is a lymphatic organ that was often thought of as an organ of mystery by the ancient Greeks. A soft, pliable lymphatic organ positioned in the anterior superior mediastinum, it does not compress or displace the adjacent structures. It is disproportionately larger in the paediatric population and then gradually regresses to 'hide' in the mediastinum as the child advances into puberty. It regresses in size under conditions of stress and may be absent in various congenital abnormalities such as DiGeorge syndrome and conditions of stress. The thymus appears in a variety of shapes and sizes on a paediatric chest radiograph and at times may be misinterpreted as pathology. This article describes the normal thymus as it appears on paediatric radiography, and addresses mimickers.

\section{Introduction}

The thymus has long been thought of as a mystical organ. The word 'thymus' is a Latin derivation from the Greek word 'thymos' that means 'warty excrescence'. For very long, the thymus was apportioned blame for a variety of childhood illnesses, based on the assumption that it compressed the airway, and was even regarded as the 'seat of Satan'. ${ }^{1}$

The thymus arises during the 6th gestational week from the 3rd and the 4th pharyngeal pouches bilaterally, as a bud-like primordium that elongates at the 7 th week, becomes cylindrical and migrates inferiorly and medially into its final position in the anterior mediastinum where the two lobes fuse..$^{2,3,4}$ It may extend superiorly to the lower pole of the thyroid, to which it is connected by the thyrothymic ligament, and an inferior extension up to the diaphragm. ${ }^{1,5}$

The thymus is a lymphatic organ that has an important role in the development of the immune system of the body, especially the T and B lymphocytes, which in turn play a major role in cellular and humoral immunity respectively.

\section{Discussion}

Despite the advances in imaging technology, the fear of radiation in children means that the chest radiograph remains the imaging modality of choice in paediatric patients. The thymus on the radiograph lies in the anterior mediastinum, overlying the pericardium, aortic arch, left innominate vein and trachea. ${ }^{4,5,6,7}$ There are numerous variations in the size and shape of the thymus on chest radiographs that are often misconstrued as pathology. A basic understanding of the anatomy and physiology of the thymus is helpful in the radiographic interpretation of its variations in chest radiography.

The thymus is extremely variable in its radiographic appearance; it widens on expiration and narrows and elongates on inspiration. ${ }^{8}$ It is a homogenous quadrilateral structure with the lateral margin being convex outward with gentle undulating margins. There is usually an increase in size in the first few months of life (Figure 1), the prominence of the thymus reflecting the health of the child. ${ }^{9}$ It is more prominent in boys than girls and decreases in size by the end of the first decade of life. ${ }^{4}$

On a frontal chest radiograph, the thymus is a prominent soft-tissue density in the superior mediastinum which appears to be inseparable from the superior cardiac margins (Figure 2a). ${ }^{8}$ The lateral view, however, confirms the opacity's location in the anterior mediastinum (Figure $2 b$ ). On the frontal view, the left lobe of the thymus produces widening of the mediastinum that usually overlaps the left pulmonary artery (Figure 3), which is occasionally visible through it. ${ }^{8}$ The anterior lateral margin often has smooth undulations from the overlying ribs and the costal cartilages (Figure 4), the so-called 'wave' or 'ripple' sign., ${ }^{4,8,9,10}$ This may be especially prominent in infants with bronchiolitis. ${ }^{9}$ A small notch (Figure 5) sometimes marks the inferior border between 


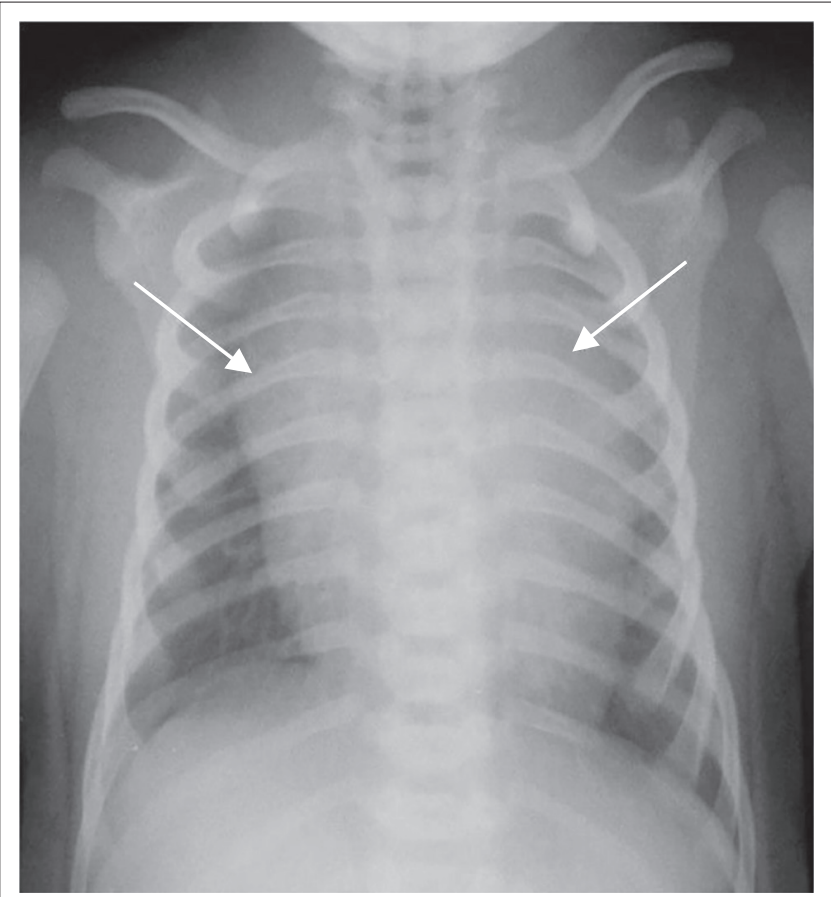

FIGURE 1: Frontal chest radiograph in an infant. A prominent thymus (arrows) gives an impression of a widened mediastinum.

the thymus and the heart. ${ }^{8,10}$ The angular corner, usually of the right lobe flattened at the right minor fissure, gives the classical 'sail sign' (Figure 6)..$^{4,8,9,11}$ A normal gland may potentially obscure anywhere from one-third to the entire left upper lobe (Figure 7); the right lobe may exhibit similar features. ${ }^{3}$

Prominence of one or more lobes can simulate cardiomegaly on frontal radiography (Figure 8) that can, however, be seen to be thymus on the lateral radiograph. Normal thymus may obscure or simulate upper lobe pneumonia (Figure 7).,7,8 Sometimes the presence of other pathologies may cause the thymus to appear abnormal, as seen in pneumomediastinum or pneumothorax where the thymus has been likened to 'angel's wings' or 'spinnaker sail' (Figure 8). Pneumothorax, when under tension, may cause displacement of the thymus, disguising it as an abnormality (Figure 9).

If radiographic findings are not conclusive, ultrasound can be used as a non-invasive modality to confirm. The normal thymus on ultrasound has an echogenicity similar to that of the liver and spleen and appears as a homogenous pliable structure that does not compress or displace adjacent structures. ${ }^{1}$

\section{Conclusion}

It is important for the radiologist and paediatrician to empirically recognise and differentiate normal thymus, its
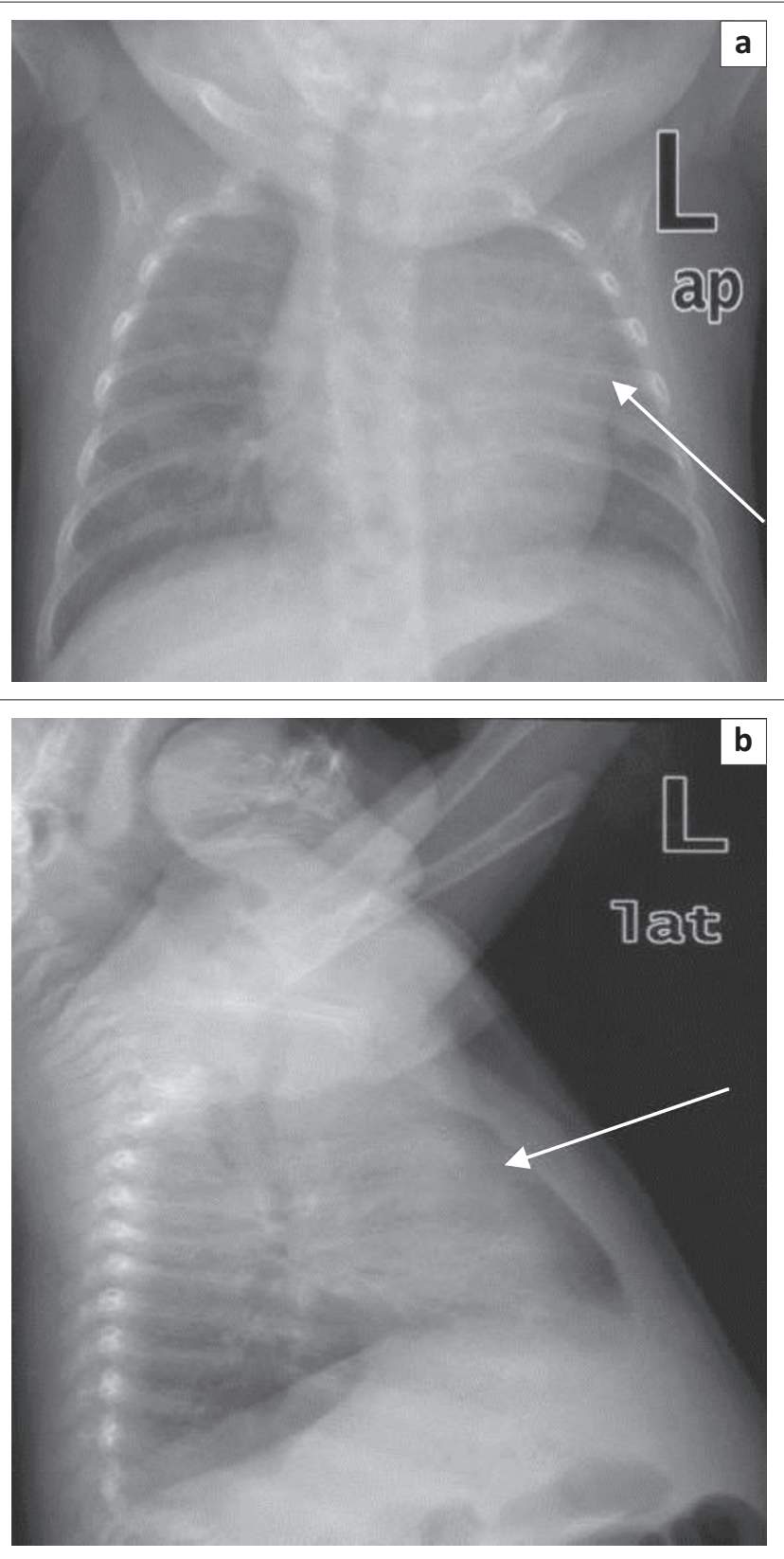

FIGURE 2: Frontal chest radiograph (a) showing apparent cardiomegaly as a result of thymus (arrow) density overlying the left heart border. A lateral view (b) identifies the opacity as a thymus (arrow) in the anterior mediastinum separate from the cardiac silhouette.

variants and mimics from pathology on a chest radiograph. A thorough knowledge of its anatomy and dynamic changes through life is essential to prevent errors in diagnosis. In cases of diagnostic confusion, imaging modalities such as ultrasound, computed tomography (CT) and positron emission tomography (PET)-CT are available and useful in differentiating thymus from other anterior mediastinal masses. 


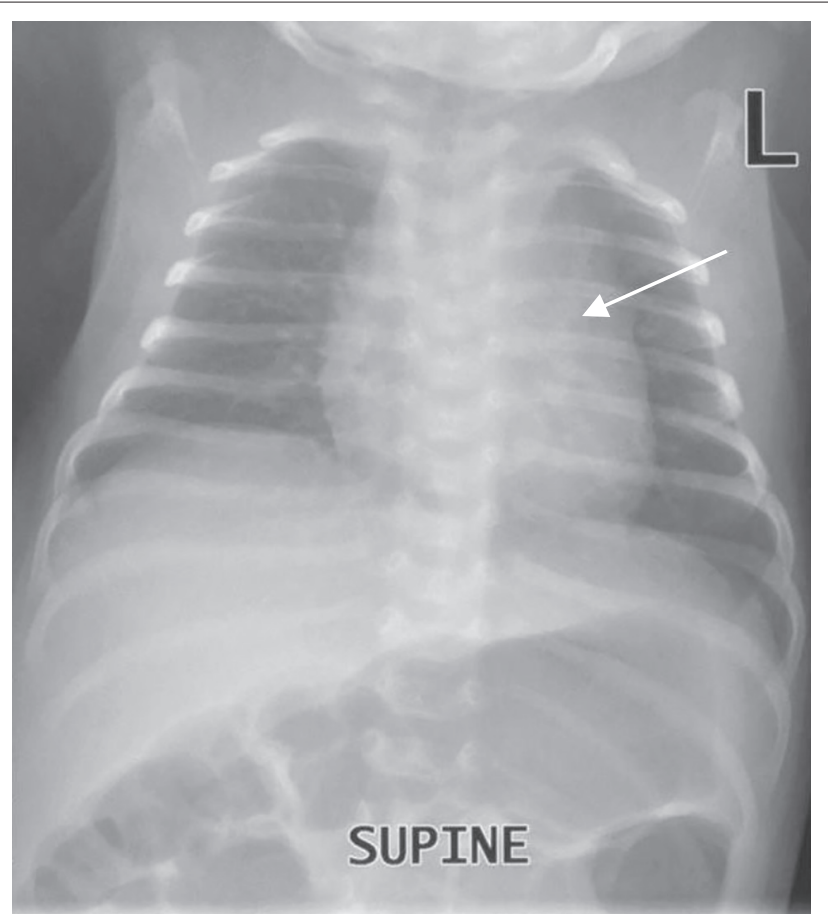

FIGURE 3: Frontal chest radiograph. The left lobe of the thymus (arrow) projects over the left hilum. The left pulmonary artery is clearly visible through the thymic shadow.

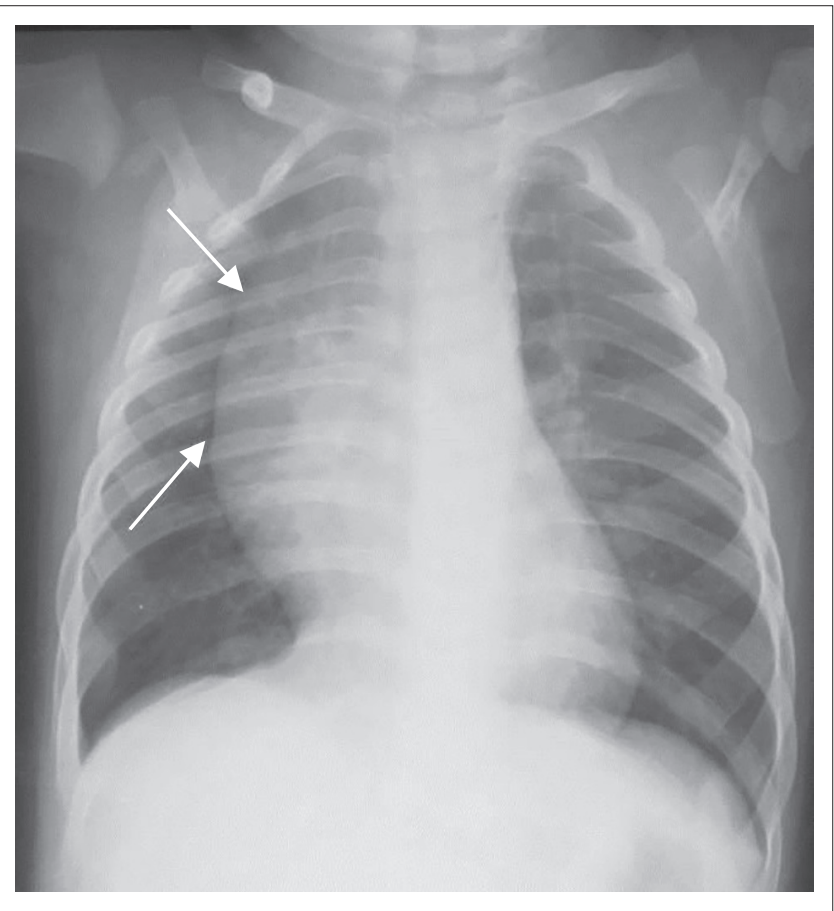

FIGURE 4: Frontal chest radiograph. Smooth undulations of the right latera margins of the thymus from the overlying ribs and cartilages, the so-called 'wave' or 'ripple' sign (arrows).

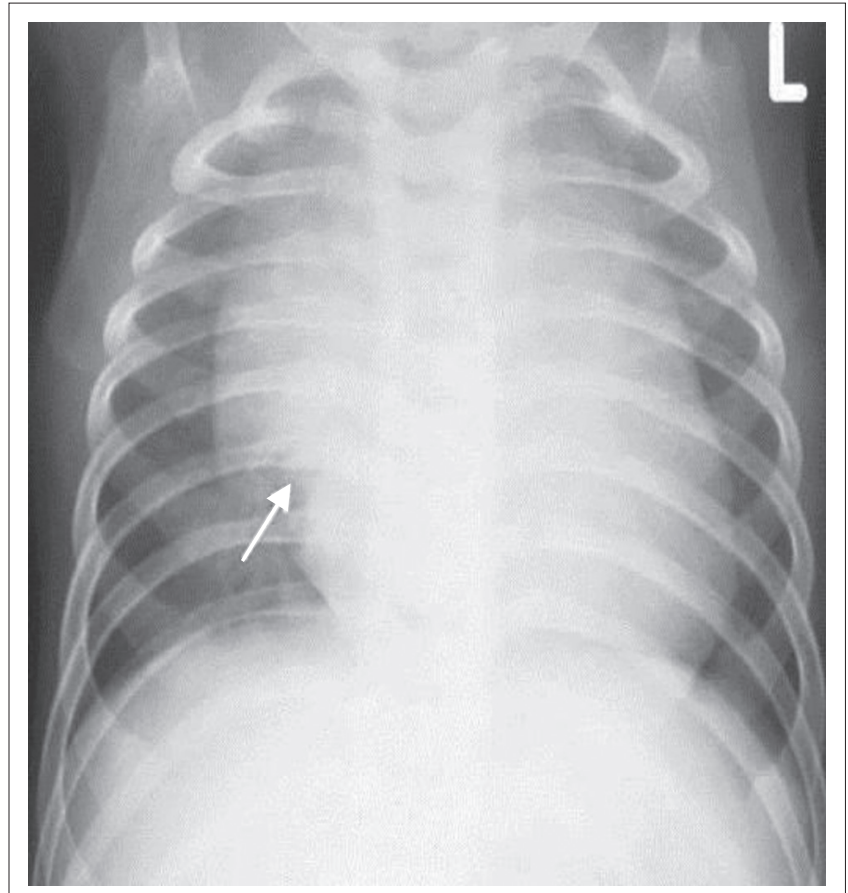

FIGURE 5: Frontal chest radiograph. There is a notch between the inferior border of the right lobe of the thymus and the heart (arrow).

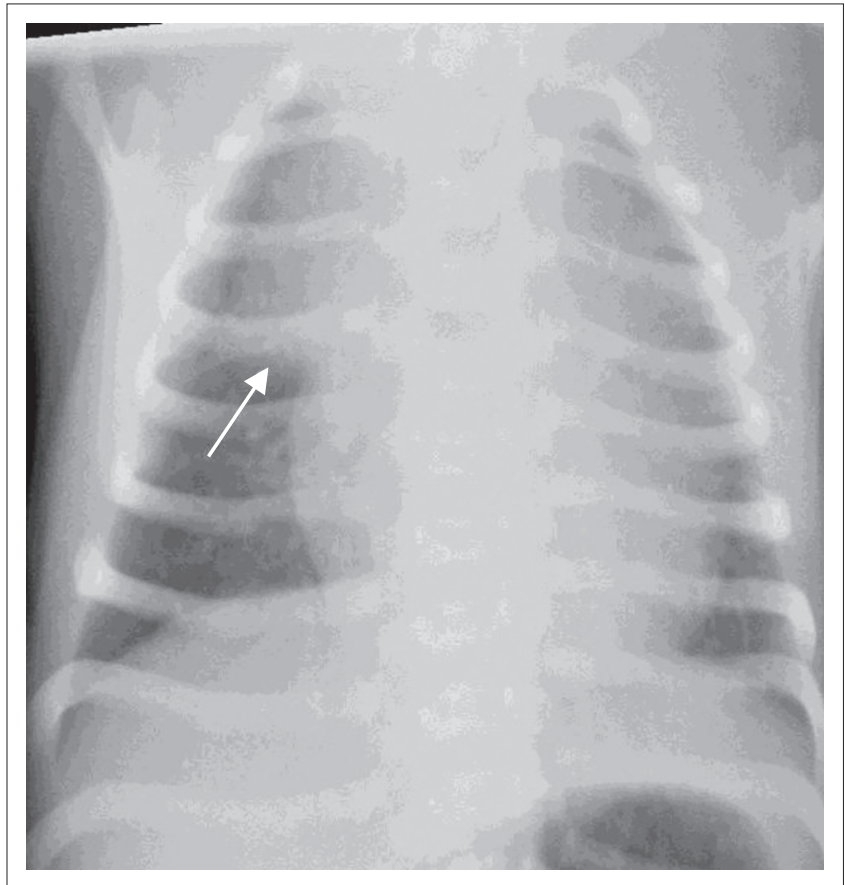

FIGURE 6: Frontal chest radiograph. A classic sail sign (arrow) formed by the right lobe of the thymus flattened at the minor fissure. 


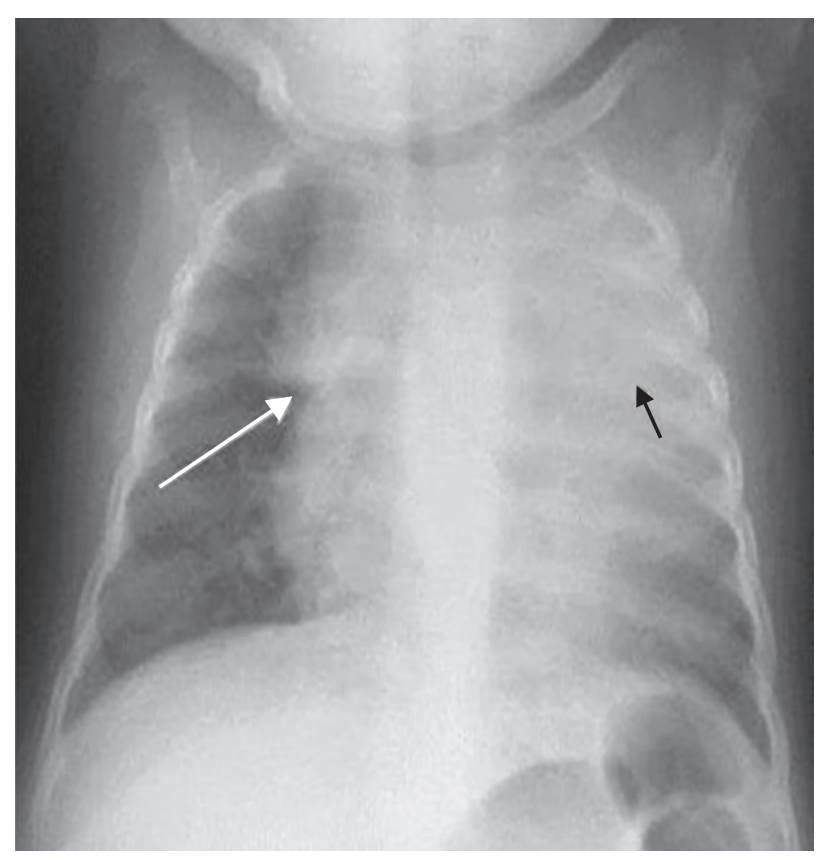

FIGURE 7: The normal thymus may obscure an entire upper lobe, as seen in this radiograph simulating pneumonia (black arrow). Note the notch sign (white arrow) on the right.

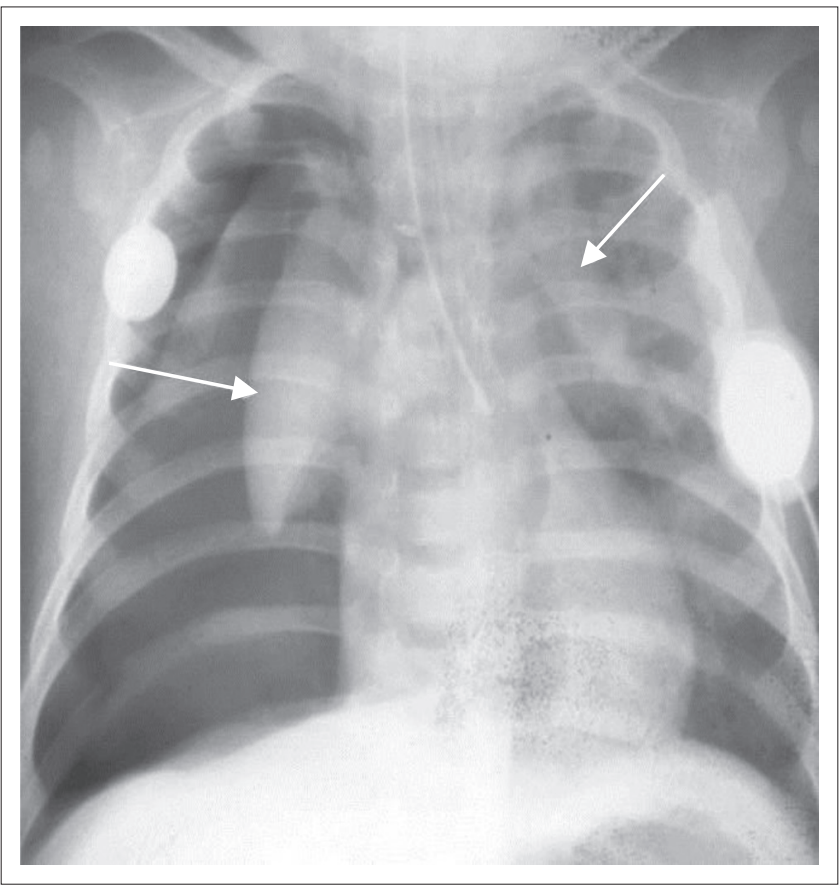

FIGURE 8: 'Angel's wing' sign (arrows) of the thymus in a patient with pneumomediastinum. The patient also has a bilateral pneumothorax.

\section{Acknowledgements}

\section{Competing interests}

The authors declare that they have no financial or personal relationships which may have inappropriately influenced them in writing this article.
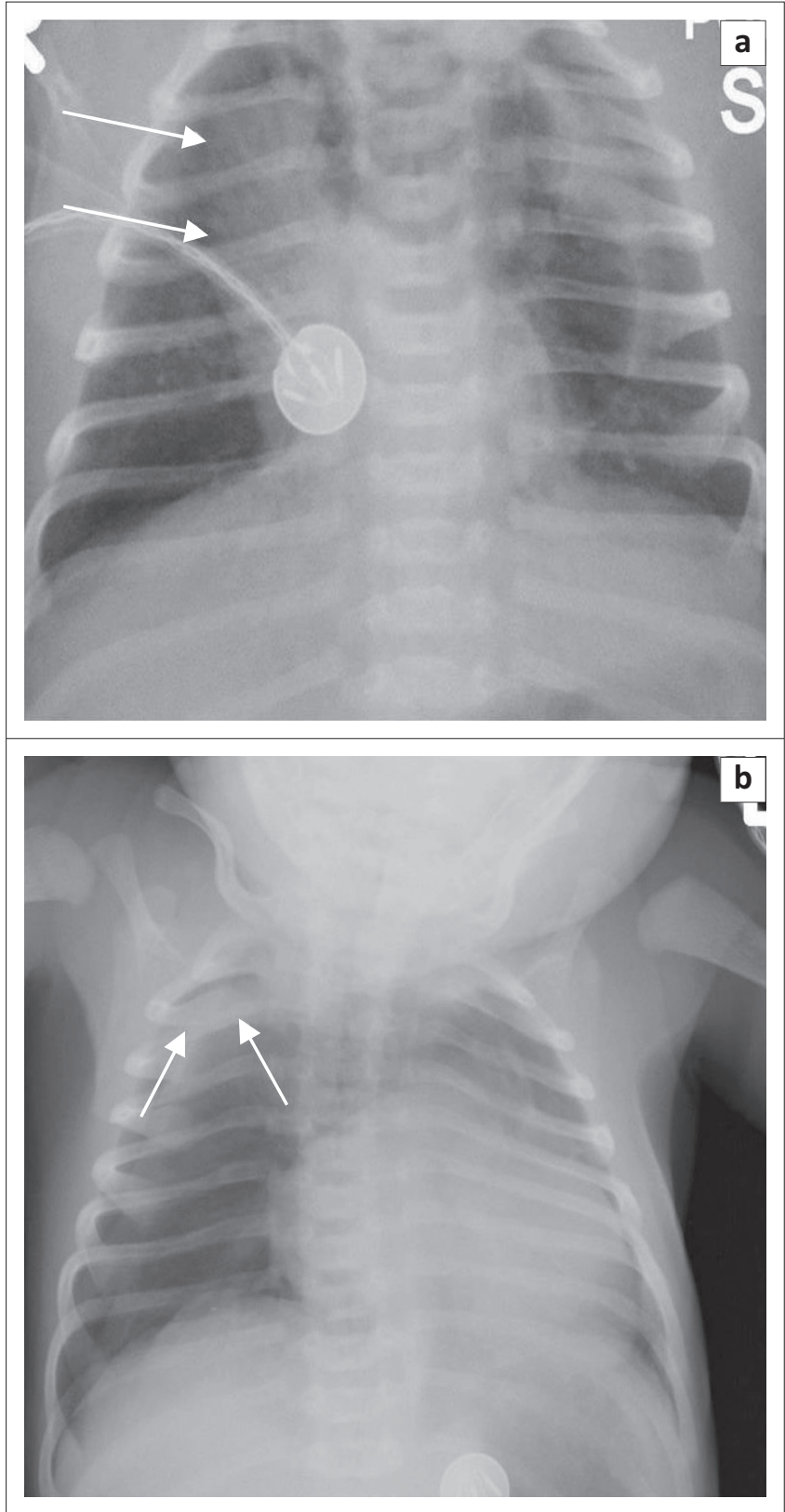

FIGURE 9: Patients with a left-sided tension pneumothorax. The thymus is displaced to the right (arrow) simulating a mediastinal mass (a). In another patient (b) with a pneumothorax, the thymus is displaced superiorly (arrows), appearing as an apical pleural abnormality.

\section{Authors' contributions}

N.K. (University of Pretoria), D.C.T. (University of Pretoria), F.S. (University of Pretoria) and I.V. (University of Pretoria), contributed equally to the writing of this manuscript.

\section{References}

1. Nasseri F, Eftekhari F. Clinical and radiologic review of normal and abnormal thymus: Pearls and pitfalls. Radiographics. 2010;30:413-428. PMID: 20228326, http://dx.doi.org/10.1148/rg.302095131

2. Nishino M, Ashiku SK, Kocher ON, Thurer RL, Boiselle PM, Hatabu H. The thymus: A comprehensive review. Radiographics. 2006;26:335-348. PMID: 16549602, http://dx.doi.org/10.1148/rg.262045213 
3. Francis IR, Glazer GM, Bookstein FL, Gross BH. The thymus: Re-examination of age-related changes in size and shape. AJR. 1985;145:249-254. PMID: 3875220, http://dx.doi.org/ 10.2214/ajr.145.2.249

4. Donelly LF, Jones BV, O'Hara SM, et al., editors. Diagnostic imaging: Pediatrics. Salt Lake City: Amirsys; 2005, p. 2 (78-79).

5. Mittal MK, Sureka B, Sinha M, Mittal A, Thukral BB. Thymic masses: A radiological review. S Afr J Radiol. 2013;17:108-111. http://dx.doi.org/10.1594/ ecr2015/C-0895

6. Gwande RS, Khurana A, Messing S, et al. Differentiation of normal thymus from anterior mediastinal lymphoma and lymphoma recurrence at pediatric PET/CT. Radiology. 2012;262:613-622. PMID: 22157202, http://dx.doi.org/10.1148/radiol 11110715
7. Federle MP, Rosado-de-Christenson ML, Woodward PJ, et al. Diagnostic and surgical imaging anatomy: Chest, abdomen, pelvis. Salt Lake City: Amirsys: 2006, p. 1 (297-298).

8. Kuhn JP, Slovis TL, O Haller J. Caffey's pediatric diagnostic imaging, vol. 1. 10th edn St Louis: Mosby; 2004, p. 1175-1184.

9. Singleton EB, Wagner ML, Dutton RV. Radiologic atlas of pulmonary abnormalities in children. Philadelphia: WB Saunders; 1971, p. 13-23.

10. Gupta AK. Normal thymus mimicking 'mediastinal mass'. Indian J Pediatr. 2009;76:1067-1068. PMID: 19907946, http://dx.doi.org/10.1007/s12098-009-0207-x

11. Arthur R. Interpretation of the paediatric chest $x$-ray. Curr Paediatr. 2003;13:438447. http://dx.doi.org/10.1016/S0957-5839(03)00089-7 